Supporting Information

\title{
Tuning the Infrared Absorption of SiC Metasurfaces by Electrically Gating Monolayer Graphene with Solid Polymer Electrolyte for \\ Dynamic Radiative Thermal Management and Sensing Applications
}

\author{
Linshuang Long, ${ }^{1}$ Xiaoyan Ying, ${ }^{1}$ Yue Yang,${ }^{1,2}$ and Liping Wang ${ }^{1, *}$ \\ ${ }^{1}$ School for Engineering of Matter, Transport \& Energy, \\ Arizona State University, Tempe, AZ 85287, USA \\ ${ }^{2}$ School of Mechanical Engineering and Automation, \\ Harbin Institute of Technology, Shenzhen, 518055, PR China \\ *Email: liping.wang@asu.edu
}

\section{Relation between graphene chemical potential and gate voltage}

The chemical potential of graphene follows

$$
\mu=\hbar\left|v_{\mathrm{F}}\right| \sqrt{\pi n}
$$

where $\left|v_{\mathrm{F}}\right|=1.1 \times 10^{6} \mathrm{~m} / \mathrm{s}$ is the Fermi velocity, and $n$ is the carrier concentration in graphene monolayer. It can be seen that a possible way to tune the chemical potential lies in varying the carrier concentration. The carrier concentration is defined by $n=q / e$, where $q$ is the charge held by the graphene layer. The relation among the gate voltage $V_{\mathrm{g}}$, chemical potential of graphene, and the charge is given by ${ }^{1}$

$$
V_{\mathrm{g}}-V_{\text {Dirac }}=\frac{\mu}{e}+\frac{q}{C_{\mathrm{g}}}
$$

where $V_{\text {Dirac }}$ is the Dirac voltage of graphene, and $C_{\mathrm{g}}$ is the geometric capacitance of the gate capacitor formed by the graphene and the electrolyte. The first item on the right-hand 
side represents a shift in the chemical potential caused by the addition of charge carriers, and the second item reflects an electrostatic potential difference between the graphene and the electrode. ${ }^{2}$

For the scenario in this study, a parallel-plate capacitor was formed at the interface between the graphene and electrolyte. The capacitance follows $C_{\mathrm{G}}=\varepsilon_{0} \varepsilon_{\mathrm{d}} / d_{\mathrm{e}}$, where $d_{\mathrm{e}}$ is the thickness of the Debye layer and $\varepsilon_{\mathrm{d}}$ is the relative permittivity of the dielectric medium. Here $\varepsilon_{\mathrm{d}}=5$ as the medium is the PEO matrix which constrains the movement of the cations. ${ }^{3}$ The thickness of the Debye layer was reported to be $\sim 1-5 \mathrm{~nm},{ }^{4}$ and $d_{\mathrm{e}}=3 \mathrm{~nm}$ is assumed here. Then we obtained a gate capacitance $C_{\mathrm{G}}=0.0148 \mathrm{~F} \mathrm{~m}^{-2}$. Combining Eq.(1) and (2), we can calculate the graphene chemical potential $\mu$ as approximately $0.2 \mathrm{eV}$ at $V_{\mathrm{g}}$ $=0 \mathrm{~V}$, and $0.8 \mathrm{eV}$ at $V_{\mathrm{g}}=5 \mathrm{~V}$.

\section{Additional measurements of tunable absorptance peaks}
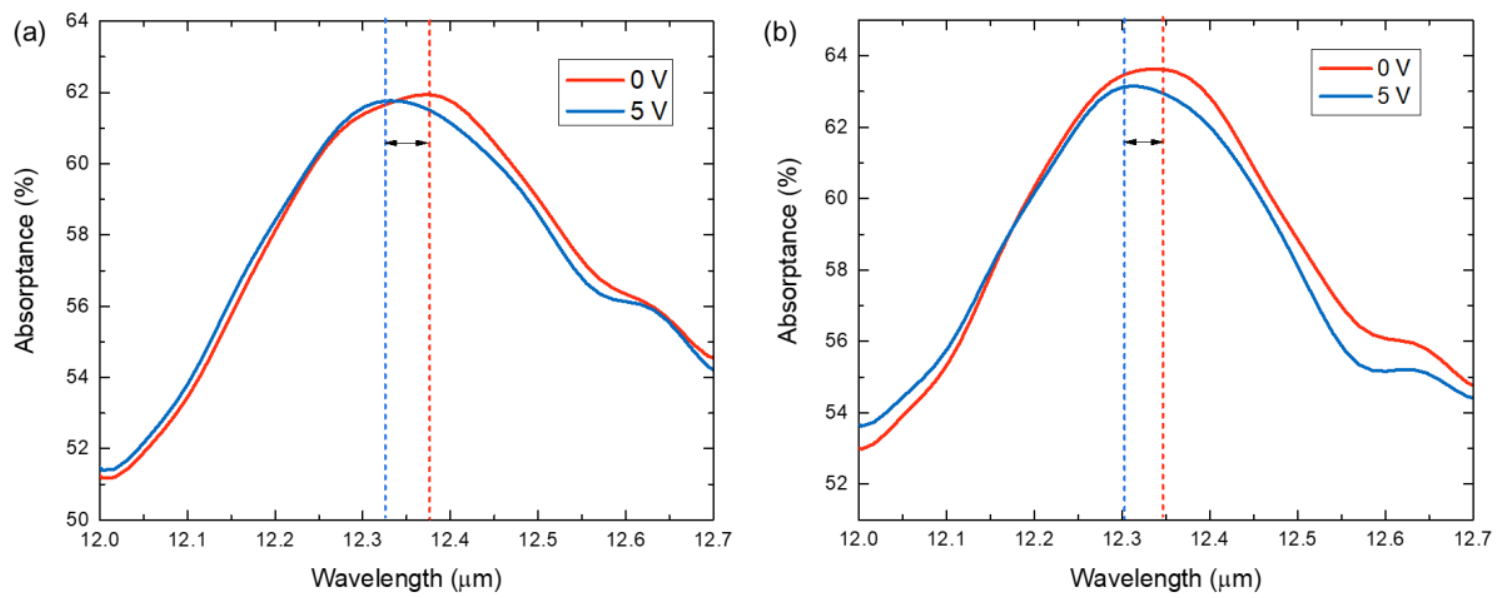

Fig. S1. Additional measurement of spectral absorptance of the metasurface at different voltages. 
Additional measurements of the spectral absorptance of the metasurface at different voltages are provided in Fig. S1 to show the repeatability. Note that the location and magnification of the peaks are slightly different for each measurement because the concentration of the electrolyte may vary each time.

\section{Relation between graphene properties and applied gate voltage}

By applying a gate voltage to the graphene and electrolyte, the absorption/emission peak of the metasurface can be tuned. The mechanism of this tunability is the change in the optical properties of graphene upon the voltage. The relation between the applied voltage and the graphene properties is explained here for better understanding of the working principle.

For a graphene monolayer, the permittivity can be described by ${ }^{5-7}$

$$
\varepsilon_{\mathrm{G}}=1+\frac{i \sigma_{\mathrm{G}}}{\omega \varepsilon_{0} t_{\mathrm{G}}}
$$

where $\sigma_{\mathrm{G}}$ is the conductivity of graphene, $\varepsilon_{0}$ is the vacuum permittivity, $\omega$ is the angular frequency, and $t_{\mathrm{G}}$ is the thickness of the monolayer. The conductivity includes two components, i.e., $\sigma_{\mathrm{G}}=\sigma_{\mathrm{D}}+\sigma_{\mathrm{I}}$, where $\sigma_{\mathrm{D}}$ and $\sigma_{\mathrm{I}}$ correspond to the intraband electronphoton scattering and interband electron transition, respectively. The two components are obtained by

$$
\sigma_{\mathrm{D}}=\frac{i}{\omega+i / \tau} \frac{2 e^{2} k_{\mathrm{B}} T}{\pi \hbar^{2}} \ln \left[2 \cosh \left(\frac{\mu}{2 k_{\mathrm{B}} T}\right)\right]
$$




$$
\sigma_{\mathrm{I}}=\frac{e^{2}}{4 \hbar}\left[G\left(\frac{\hbar \omega}{2}\right)+i \frac{4 \hbar \omega}{\pi} \int_{0}^{\infty} \frac{G(\xi)-G(\hbar \omega / 2)}{(\hbar \omega)^{2}-4 \xi^{2}} d \xi\right]
$$

where $e$ is the electron charge, $\hbar$ is the reduced Planck constant, $k_{\mathrm{B}}$ is the Boltzmann constant, $\mu$ is the chemical potential, $\tau$ is the relaxation time, $T$ is the temperature, and $G(\xi)=\sinh \left(\xi / k_{\mathrm{B}} T\right) /\left[\cosh \left(\mu / k_{\mathrm{B}} T\right)+\cosh \left(\xi / k_{\mathrm{B}} T\right)\right]$. Here $\tau$ and $T$ are taken as constants, and their values are $10^{-13} \mathrm{~s}$ and $300 \mathrm{~K}$, respectively. ${ }^{8}$ As a result, the only variable that determines the conductivity of graphene is the chemical potential, which is a function of gate voltage as per the previous section. After all, the relation between the permittivity of graphene and applied voltage can be established by combining Eq. (1) (5).

\section{References}

1. Yan, R.; Zhang, Q.; Li, W.; Calizo, I.; Shen, T.; Richter, C. A.; Hight-Walker, A. R.; Liang, X.; Seabaugh, A.; Jena, D.; Grace Xing, H.; Gundlach, D. J.; Nguyen, N. V., Determination of graphene work function and graphene-insulator-semiconductor band alignment by internal photoemission spectroscopy. Appl Phys Lett 2012, 101 (2), 022105.

2. $\quad$ Das, A.; Pisana, S.; Chakraborty, B.; Piscanec, S.; Saha, S. K.; Waghmare, U. V.; Novoselov, K. S.; Krishnamurthy, H. R.; Geim, A. K.; Ferrari, A. C.; Sood, A. K., Monitoring dopants by Raman scattering in an electrochemically top-gated graphene transistor. Nature nanotechnology 2008, 3 (4), 210-5.

3. Boyd, R. H., The dielectric constant of lamellar semicrystalline polymers. Journal of Polymer Science: Polymer Physics Edition 1983, 21 (4), 505-514.

4. $\quad$ Lu, C.; Fu, Q.; Huang, S.; Liu, J., Polymer Electrolyte-Gated Carbon Nanotube Field-Effect Transistor. Nano letters 2004, 4 (4), 623-627.

5. Falkovsky, L. A.; Varlamov, A. A., Space-time dispersion of graphene conductivity. The European Physical Journal B 2007, 56 (4), 281-284.

6. Falkovsky, L. A., Optical properties of graphene. Journal of Physics: Conference Series 2008, 129, 012004.

7. Falkovsky, L. A.; Pershoguba, S. S., Optical far-infrared properties of a graphene monolayer and multilayer. Physical Review B 2007, 76 (15), 153410.

8. Lim, M.; Lee, S. S.; Lee, B. J., Near-field thermal radiation between graphenecovered doped silicon plates. Opt Express 2013, 21 (19), 22173-85. 